\title{
THE BOREL CARDINALITY OF LASCAR STRONG TYPES
}

\author{
ITAY KAPLAN, BENJAMIN MILLER, AND PIERRE SIMON
}

\begin{abstract}
We show that if the restriction of the Lascar equivalence relation to a KP-strong type is non-trivial, then it is non-smooth (when viewed as a Borel equivalence relation on an appropriate space of types).
\end{abstract}

\section{INTRODUCTION}

Notions of strong type play an important role in the study of first-order theories. A strong type (over $\emptyset$ ) is a class of an automorphism-invariant equivalence relation on $\mathfrak{C}^{\alpha}$ which is bounded (i.e., the quotient has small cardinality) and refines equality of types. The phrase "strong type" by itself often refers to a Shelah strong type, which is simply a type over the algebraic closure of $\emptyset$ (in $T^{\mathrm{eq}}$ ). In other words, two sequences have the same Shelah strong type if they are equivalent with respect to every definable equivalence relation with finitely many classes. Refining this is the notion of $K P$ strong type $\left(\equiv_{K P}^{\alpha}\right)$, in which two sequences are equivalent if they are equivalent with respect to every bounded type-definable equivalence relation. The KP strong type can also be characterized as the finest notion of strong type for which the corresponding quotient is a compact Hausdorff space. Finally, the Lascar strong type $\left(\equiv_{L}^{\alpha}\right)$ is the finest notion of strong type. The classes of $\equiv_{L}^{\alpha}$ coincide with the connected components of the Lascar graph on $\mathfrak{C}^{\alpha}$, in which two sequences are neighbors if they lie along an infinite indiscernible sequence. The Lascar distance $d$ is the associated graph distance. All of this is explained in detail in Subsection 1.1.

In [New03, Newelski established the following fundamental facts:

Fact 1.1. New03] Suppose that $T$ is a complete first-order theory and $\alpha$ is an ordinal.

(1) A Lascar strong type is type definable iff it has finite diameter.

(2) If $Y$ is an $\equiv_{L}^{\alpha}$-invariant closed set, contained in some $p \in S_{\alpha}(\emptyset)$, on which every $\equiv_{L}^{\alpha}$-class has infinite diameter, then $Y$ contains at least $2^{\aleph_{0}}$-many $\equiv_{L}^{\alpha}$-classes.

(3) Lascar strong types of unbounded diameter are not $G_{\delta}$ sets (when viewed as subsets of an appropriate space of types, as explained in Subsection [1.4).

(4) If $T$ is small (i.e., $T$ is countable and the number of finitary types over $\emptyset$ is countable), then $\equiv_{L}^{n}=\equiv_{K P}^{n}$ for all $n<\omega$ (i.e., the two notions of type agree on finite sequences). 
As opposed to Shelah and KP strong types, the space of Lascar strong types does not come equipped with a Hausdorff topology. It is therefore unclear to what category this quotient belongs. In KPS12, the authors suggest viewing it through the framework of descriptive set theory (this idea was already mentioned in [CLPZ01]). They formally interpret the notion of equality of Lascarstrong types as a Borel equivalence relation over a compact Polish space, and then consider the position of this relation in the Borel reducibility hierarchy.

Given two Polish spaces $X$ and $X^{\prime}$ and two Borel equivalence relations $E$ and $E^{\prime}$ respectively on $X$ and $X^{\prime}$, we say that $E$ is Borel reducible to $E^{\prime}$ if there is a Borel map $f$ from $X$ to $X^{\prime}$ such that $x E y \Longleftrightarrow f(x) E^{\prime} f(y)$ for all $x, y \in X$. Two relations are Borel bi-reducible if each is Borel reducible to the other. The quasi-order of Borel reducibility is a well-studied object in descriptive set theory, and enjoys a number of remarkable properties. One of them is given by the Harrington-Kechris-Louveau dichotomy, which asserts that a Borel equivalence relation is either smooth (Borel reducible to equality on $2^{\omega}$ ) or at least as complicated as $\mathbb{E}_{0}$ (eventual equality on $\left.2^{\omega}\right)$. This is explained in detail in Subsections 1.2 and 1.2 ,

In this paper, we provide the following solution to the main conjecture of [KPS12].

Main Theorem A. [Simplified version] Suppose that $T$ is a complete countable first-order theory. If $\equiv_{L}$ does not coincide with $\equiv_{K P}$, then $\equiv_{L}$ is not smooth.

We will actually prove a slightly stronger result (see Theorem 4.12). Our proof will not make use of Fact 1.1 which we will recover (for countable $T$ ) as a corollary.

Let us say a few words about our method. In [NP06, Newelski and Petrykowski introduce the notion of weakly generic types for definable groups. An analog for groups of automorphisms was used in Pelaez's thesis Pel08 to give an alternate proof of Fact 1.1(1). We follow this lead in our own proof.

A consequence of the proof of an early special case of the Harrington-Kechris-Louveau dichotomy theorem is that if $X$ is a Polish space, $G$ is a Polish group acting continuously on $X$, and the orbit equivalence relation $E_{G}$ is $F_{\sigma}$, then either $E_{G}$ is smooth or $\mathbb{E}_{0}$ can be continuously embedded into $E_{G}$. This is related to BK96, Theorem 3.4.5], which gives a sufficient condition for embedding $\mathbb{E}_{0}$ in an equivalence relation induced by a group action (namely that there is a dense orbit and that $E_{G}$ is meager).

As a corollary of the latter result, we establish a sufficient criterion for embedding $\mathbb{E}_{0}$ into an equivalence relation $E$ whose classes are each equipped with a metric. Roughly speaking, the group $G$ of homeomorphisms of the Cantor space whose graphs are contained in $\mathbb{E}_{0}$ acts in a sufficiently rich fashion that it can move any element to another which is arbitrarily close in the topological sense, but far away in the sense of the metric associated with the class. We show that if a similar property holds for $E$ then one can embed $\mathbb{E}_{0}$ into $E$. 
Assuming that there is a Lascar-strong type of unbounded diameter, it is thus natural to try to find a type $p$ whose orbit under the group of Lascar-strong automorphisms is also sufficiently rich. When $T$ is countable, we construct such a type formula-by-formula. At each stage, we must make sure that we still have room to go on, namely, that the partial type has many images which are at large Lascar distance from each other. To this end, we make sure that the type stays weakly generic. We actually need a slightly stronger property which we call "properness".

Main Theorem $\mathrm{A}$ does not seem to be enough to deduce Fact 1.1 for uncountable theories. However, we adapt our argument to also take care of this case. This is Main Theorem B, For uncountable languages, the space of types is no longer a Polish space, so we do not state the theorem in terms of Borel cardinality. Apart from that, the result is essentially the same as for countable theories. In particular, it implies Newelski's theorem. In order to prove it, we will need a little bit more from the descriptive set theoretic side, namely, the notion of (strong) Choquet space. This is used to replace completeness. In fact, eventually we deal with a non-Hausdorff space. (A non-Hausdorff space will arise as the space of types over a model $M$ with the topology induced by a countable sub-language $L^{\prime}$ of $L$ and a corresponding countable model $M^{\prime}$.)

Organization of the paper. We have made an effort to keep this paper self-contained and accessible to model theorists and descriptive set theorists alike. Thus we start by giving all of the required definitions from both sides. In Section 2, we state a set theoretic criterion for non-smoothness. In Section 3, we treat a special case of the main theorem, where $T$ is small (hence Lascar strong types coincide with KP strong types on finite tuples) and $\alpha$ is infinite. Although this result will not be used to prove the general case, we thought it worthwhile to include, as the proof is considerably simpler and gives insight into the general case. In Section 4, we prove Main Theorem A for all countable theories. Finally, in section [5, we prove Main Theorem B thereby taking care of the general case.

1.1. Model-theoretic preliminaries. Let $T$ be any complete first order theory. The theory $T$ may be many sorted, but for the simplicity of the presentation one may assume that it is one sorted. We recall some basic notions. We fix a sequence of variables $\left\langle v_{i}\right| i \in$ ord $\rangle$. For the rest of this section, $\alpha$ will be some ordinal.

Definition 1.2. Suppose $M \models T, A \subseteq M$. Let $L_{\alpha}(A)$ the set of formulas in variables $\left\langle v_{i} \mid i<\alpha\right\rangle$ with parameters from $A$ - the set of formulas over $A$. An $\alpha$-type over $A$ (sometimes a partial $\alpha$-type over $A$ ) is a consistent subset of $L_{\alpha}(A)$. An $\alpha$-type $p$ over $A$ is called complete if for any formula $\varphi \in L_{\alpha}(A),\{\varphi, \neg \varphi\}$ intersects $p$. We denote by $S_{\alpha}(A)$ the space of all complete $\alpha$-types over $A$. For a tuple $a \in M^{\alpha}$, let

$$
\operatorname{tp}(a / A)=\left\{\varphi \in L_{\alpha}(A) \mid M \models \varphi(a)\right\} .
$$


We write $a \equiv_{A} b$ for $\operatorname{tp}(a / A)=\operatorname{tp}(b / A)$. If $A=\emptyset$, we omit it. If $p$ is an $\alpha$-type over $A$ and $\operatorname{tp}(a / A) \supseteq p$ we write $a \models p$. We say that $p$ is realized in $M$ if there exists some $a \in M^{\alpha}$ such that $a \models p$.

We sometimes write $p(x)$ (respectively $\varphi(x)$ ) when we want to stress that the free variables of $p$ (respectively $\varphi$ ) are contained in the tuple of variables $x$.

Remark 1.3. The set $S_{\alpha}(A)$ is naturally a compact Hausdorff topological space, the Stone space of $\alpha$-types over $A$, with clopen basis $\left\{[\varphi] \mid \varphi \in L_{\alpha}(A)\right\}$, where $[\varphi]=\left\{p \in S_{\alpha}(A) \mid \varphi \in p\right\}$.

Definition 1.4. For a cardinal $\kappa$, a model $M \models T$ is called $\kappa$-saturated when for all sets $A \subseteq M$ of cardinality $<\kappa$ all types in $S_{1}(A)$ are realized in $M$. The model $M$ is $\kappa$-homogeneous when for all $\alpha<\kappa$ and $a, b \in M^{\alpha}$ if $a \equiv b$ then there is an automorphism of $M$ mapping $a$ to $b$.

Recall that the cardinality of $T,|T|$, is identified with the cardinality of the set of formulas in $T$.

Fact 1.5. Hod93, Theorem 10.2.1] For any cardinal $\kappa \geq|T|$, and any model $M \models T$ there exists a $\kappa$-saturated, $\kappa$-homogeneous model $N \succ M$ of $T$.

Fix some $\kappa \geq\left(2^{|T|}\right)^{+}$. We denote by $\mathfrak{C}$ a $\kappa$-saturated, $\kappa$-homogeneous model of $T$. In model theory, this is usually referred to as the "monster model" of $T$ (and it is often harmless to assume in addition that $\mathfrak{C}$ is $|\mathfrak{C}|$-saturated and is very big). The convention is that all sets of parameters and tuples we deal with are small, that is, of cardinality $<\kappa$, and that they are all contained in $\mathfrak{C}$. Similarly all small models are assumed to be elementary substructures of $\mathfrak{C}$.

Recall that for $A \subseteq \mathfrak{C}$, Aut $(\mathfrak{C} / A)$ denotes the group of automorphisms of $\mathfrak{C}$ which fix $A$ pointwise.

Definition 1.6. Let $A \subseteq \mathfrak{C}$ a small set. A set $X \subseteq \mathfrak{C}^{\alpha}$ is called $A$-type-definable (or type-definable over $A$ ) if it is empty or there is an $\alpha$-type $p$ over $A$ such that

$$
X=\left\{a \in \mathfrak{C}^{\alpha} \mid a \models p\right\} .
$$

It is $A$-invariant (or invariant over $A$ ) when for all $\sigma \in \operatorname{Aut}(\mathfrak{C} / A), \sigma^{\alpha}(X)=X$ (usually we omit $\alpha$ from this notation). When $A$ is omitted, it is understood that $A=\emptyset$.

We define a "topology" on subsets of $\mathfrak{C}^{\alpha}$.

Definition 1.7. Call a subset $X \subseteq \mathfrak{C}^{\alpha}$ pseudo closed if $X$ is type definable over some small set. A pseudo open set is a complement of a pseudo closed set. Pseudo $G_{\delta}$ sets and pseudo $F_{\sigma}$ sets are defined in the obvious way.

By saturation $\mathfrak{C}^{\alpha}$ is pseudo compact in the sense that any small intersection of non-empty pseudo closed sets is non-empty. This why we often say "by compactness", instead of "by saturation". 
Remark 1.8. By compactness, for a small set $A \subseteq \mathfrak{C}$, the map $r_{\alpha, A}: \mathfrak{C}^{\alpha} \rightarrow S_{\alpha}(A)$ defined by $a \mapsto \operatorname{tp}(a / A)$ is pseudo closed, in the sense that it sends pseudo closed sets to closed sets (in the Stone topology). So $r_{\alpha, A}$ maps pseudo $F_{\sigma}$ subsets of $\mathfrak{C}^{\alpha}$ to $F_{\sigma}$ subsets of $S_{\alpha}(A)$.

We also recall the notion of an indiscernible sequence:

Definition 1.9. Let $A$ be a small set. Let $(I,<)$ be some linearly ordered set. A sequence $\bar{a}=\left\langle a_{i} \mid i \in I\right\rangle \in\left(\mathfrak{C}^{\alpha}\right)^{I}$ is called A-indiscernible (or indiscernible over $A$ ) if for all $n<\omega$, every increasing $n$-tuple from $\bar{a}$ realizes the same type over $A$. When $A$ is omitted, it is understood that $A=\emptyset$.

An easy but very important fact about indiscernible sequences is that they exist.

Fact 1.10.

(1) [TZ12, Lemma 5.1.3] Let $\left(I,<_{I}\right),\left(J,<_{J}\right)$ be small linearly ordered sets, and let $A$ be some small set. Suppose $\bar{b}=\left\langle b_{j} \mid j \in J\right\rangle$ is some sequence of elements from $\mathfrak{C}^{\alpha}$. Then there exists an indiscernible sequence $\bar{a}=\left\langle a_{i} \mid i \in I\right\rangle \in\left(\mathfrak{C}^{\alpha}\right)^{I}$ such that:

- For any $n<\omega$ and $\varphi \in L_{\alpha \cdot n}$, if $\mathfrak{C} \models \varphi\left(b_{j_{0}}, \ldots, b_{j_{n-1}}\right)$ for every $j_{0}<_{J} \ldots<_{J} j_{n-1}$ from $J$ then $\mathfrak{C} \models \varphi\left(a_{i_{0}}, \ldots, a_{i_{n-1}}\right)$ for every $i_{0}<_{I} \ldots<_{I} i_{n-1}$ from $I$.

(2) [Ker07, proof of Proposition 3.1.4] If $M$ is a small model and $a \equiv_{M} b$, then there is an indiscernible sequence $\bar{c}=\left\langle c_{i} \mid i<\omega\right\rangle$ such that both $a \frown \bar{c}$ and $b \frown \bar{c}$ are indiscernible.

Point (1) in Fact 1.10 is proved using Ramsey's theorem and compactness, while (2) is proved with ultrafilters.

Definition 1.11. An equivalence relation $E$ on a set $X$ is called bounded if $|X / E|<\kappa$.

Remark 1.12. By saturation and homogeneity, every invariant set is a union of types over $\emptyset$. So by saturation if $E$ is an invariant equivalence relation with an invariant domain $X \subseteq \mathfrak{C}^{\alpha}$, it makes sense to consider $E$ in any monster model. When $E$ is bounded, and $|\alpha| \leq|T|$ then $|X / E| \leq 2^{|T|}$. To see that, let $M \models T$ be of size $|T|$. If $a, b \in X$ and $a \equiv_{M} b$ then $(a, b) \in E$, since otherwise, by Fact 1.10 (2) and saturation, we may assume that $\langle a, b\rangle$ starts an indiscernible sequence of length $\kappa$. By homogeneity, any two elements in it are not $E$-equivalent. Now the result follows from the fact that $\left|S_{\alpha}(M)\right| \leq 2^{|T|}$. It is now easy to see that if $\mathfrak{C}^{\prime} \succ \mathfrak{C}$ is another monster model then every $E$-class in $\mathfrak{C}^{\prime}$ intersects $\mathfrak{C}$, so there are no "new" classes.

We come to the central definition.

Definition 1.13. The Lascar graph on $\mathfrak{C}^{\alpha}$ is the set $G_{\alpha}$ of pairs $(a, b)$ of distinct elements of $\mathfrak{C}^{\alpha}$ which lie along an infinite indiscernible sequence. The Lascar metric $d_{\alpha}$ is the metric associated with this graph. Let $\equiv_{L}^{\alpha}$ denote the equivalence relation on $\mathfrak{C}^{\alpha}$ whose classes coincide with the 
connected components of $G_{\alpha}$. The Lascar strong type of a tuple $a \in \mathfrak{C}^{\alpha}$ is its $\equiv_{L}^{\alpha}$-class. We will omit $\alpha$ from the notation when it is clear from context.

Remark 1.14. By Fact1.10 (2), it follows that for $a, b \in \mathfrak{C}^{\alpha}$ and $M \prec \mathfrak{C}$, if $a \equiv_{M} b$ then $d(a, b) \leq 2$.

Fact 1.15. (see e.g., Ker07, Proposition 3.1.4]) The relation $\equiv_{L}^{\alpha}$ is the finest bounded invariant equivalence relation on $\mathfrak{C}^{\alpha}$.

Proof (sketch.) If $E$ is some bounded invariant equivalence relation on $\mathfrak{C}^{\alpha}$ and $d_{\alpha}(a, b) \leq 1$, then as in Remark 1.12, $(a, b) \in E$. Similarly, $\equiv_{L}^{\alpha}$ is bounded since it is bounded by $\left|S_{\alpha}(M)\right|$ for any model $M \models T$.

Definition 1.16. The group of Lascar strong automorphisms of $\mathfrak{C}$ is the group generated by automorphisms $\sigma$ of $\mathfrak{C}$ for which there is a small model $M \prec \mathfrak{C}$ fixed pointwise by $\sigma$, i.e., the group

$$
\text { Aut } f_{L}(\mathfrak{C})=\langle\sigma \in \operatorname{Aut}(\mathfrak{C} / M) \mid M \prec \mathfrak{C}\rangle
$$

Fact 1.17. (see e.g., Ker07, Section 3.1])

(1) The group of Lascar strong automorphisms is a normal subgroup of Aut ( $\mathfrak{C})$. It consists of all automorphisms that fix all Lascar strong types (of any length).

(2) The Lascar strong type equivalence relation is the orbit equivalence relation of the group of Lascar strong automorphisms.

(3) If $\sigma$ is a Lascar strong automorphism, then there is some $m<\omega$ such that for any tuple $c$ (of any length), $d(c, \sigma(c)) \leq m$. In this case we say that $m$ bounds $\sigma$.

Remark 1.18. Suppose $a, b \in \mathfrak{C}^{\alpha}$ and $d_{\alpha}(a, b) \leq n$. Then there is a Lascar strong automorphism $\sigma$ of $\mathfrak{C}$ bounded by $2 n$ such that $\sigma(a)=b$.

Proof. (of Remark 1.18) It is enough to establish it in the case $d(a, b) \leq 1$ : if $d(a, b) \leq n$, then there are $c_{0}, \ldots, c_{n}$ with $a=c_{0}, c_{n}=b$ and $d\left(c_{i}, c_{i+1}\right) \leq 1$ for all $i<n$. For each $i<n$, we find some $\sigma_{i}$ bounded by 2 that maps $c_{i}$ to $c_{i+1}$. Let $\sigma=\sigma_{n-1} \circ \ldots \circ \sigma_{0}$.

So suppose $I=\left\langle a_{i}\right| i\langle\omega\rangle$ is an indiscernible sequence that starts with $a_{0}=a, a_{1}=b$. Let $M$ be a model of size $|T|$. By saturation we can extend the sequence $I$ to length $\left(2^{|T|}\right)^{+}$. So there must be two elements in $I$ that have the same type over $M$. By indiscernibility and homogeneity, there is some model $M^{\prime}$ such that $a \equiv_{M^{\prime}} b$.

The remark now follows from Remark 1.14,

We also recall the notion of KP strong type:

Definition 1.19. Let $\equiv_{K P}^{\alpha}$ denote the finest bounded type-definable equivalence relation on $\mathfrak{C}^{\alpha}$.



${ }^{1}$ KP stands for Kim-Pillay. This notation was introduced by Hrushovski in [Hru98]. 
Fact 1.20. [Cas11, Proposition 15.25] Let $X$ be any type-definable subset of $\mathfrak{C}^{\alpha}$.

(1) The restriction $\equiv_{L}^{\alpha} \uparrow X$ of $\equiv_{L}^{\alpha}$ to $X$ is the finest bounded invariant equivalence relation on realizations of $X$.

(2) The restriction $\equiv_{K P}^{\alpha} \uparrow X$ of $\equiv_{K P}^{\alpha}$ is the finest bounded type-definable equivalence relation on realizations of $p$.

Remark 1.21. By saturation and homogeneity if $X \subseteq \mathfrak{C}^{\alpha}$ is type-definable over some small set $B$ and invariant over another small set $A$, then it is type-definable over $A$. It follows that if $K \subseteq \mathfrak{C}^{\alpha}$ is a KP strong type, and for some $a \in K,[a]_{\equiv_{L}^{\alpha}}$ is pseudo closed, then $\equiv_{L}^{\alpha} \uparrow K$ is trivial. Indeed, it is type-definable over $a$ so there is a type $\pi(x, y)$ such that $\pi(x, a)$ defines $[a]_{\equiv_{L}}$. Let $p(x)=\operatorname{tp}(a / \emptyset)$. Then $\equiv_{L}^{\alpha} \uparrow p$ is defined by: $x \equiv_{L}^{\alpha} y$ iff $\pi(x, y)$. Fact 1.20 implies that $\equiv_{L}^{\alpha} \uparrow p=\equiv_{K P}^{\alpha}\left\lceil p\right.$, so $\equiv_{L}^{\alpha} \uparrow K$ is trivial.

Definition 1.22. Let $Y \subseteq \mathfrak{C}^{\alpha}$ be closed under $\equiv_{L}^{\alpha}$. We say that $Y$ is $d$-bounded if there is some $n<\omega$ such that $a \equiv_{L}^{\alpha} b$ iff $d(a, b) \leq n$ for all $a, b \in Y$.

Remark 1.23. For a set of parameters $A$, Lascar distance over $A$, Lascar strong type over $A, K P$ strong type over $A$, etc., are the parallel notions for $T_{A}$ : the complete theory of the structure $\mathfrak{C}_{A}$ which is just $\mathfrak{C}$ after naming all elements from $A$. All the facts above hold for $A$ with the obvious adjustments.

1.2. Preliminaries on Borel equivalence relations. Here we give the basic facts about Borel equivalence relations.

Definition 1.24. Suppose $X$ and $Y$ are Polish spaces, and $E$ and $F$ are Borel equivalence relations on $X$ and $Y$. We say that a function $f: X \rightarrow Y$ is a reduction of $E$ to $F$ if for all $x_{0}, x_{1} \in X$, $\left(x_{0}, x_{1}\right) \in E$ iff $\left(f\left(x_{0}\right), f\left(x_{1}\right)\right) \in F$.

(1) We say that $E$ is Borel reducible to $F$, denoted by $E \leq_{B} F$, when there is a Borel reduction $f: X \rightarrow Y$ of $E$ to $F$.

(2) We say that $E$ is continuously reducible to $F$, denoted by $E \sqsubseteq_{c} F$, when there is a continuous injective reduction $f: X \rightarrow Y$ of $E$ to $F$.

(3) We say that $E$ and $F$ are Borel bi-reducible, denoted by $E \sim_{B} F$, when $E \leq_{B} F$ and $F \leq_{B} E$.

(4) We write $E<_{B} F$ to mean that $E \leq_{B} F$ but $E \varkappa_{B} F$.

Example 1.25. For a Polish space $X$, the relations $\Delta(X)$ denotes equality on $X$. Then $\Delta(1)<_{B}$ $\Delta(2)<_{B} \ldots<_{B} \Delta(\omega)<_{B} \Delta\left(2^{\omega}\right)$.

Definition 1.26. We say that $E$ is smooth iff $E \leq_{B} \Delta\left(2^{\omega}\right)$. 
Note that being smooth is equivalent to the existence of "separating Borel sets," i.e., Borel sets $B_{i} \subseteq X$ such that $x E y$ iff for all $i<\omega, x \in B_{i}$ iff $y \in B_{i}$.

Fact 1.27. Sil80 (Silver dichotomy) For all Borel equivalence relations $E, E \leq_{B} \Delta(\omega)$ or $\Delta\left(2^{\omega}\right) \sqsubseteq_{c} E$. It follows that $\Delta\left(2^{\omega}\right)$ is the successor of $\Delta(\omega)$.

Proposition 1.28. Closed equivalence relations are smooth.

Proof. Suppose $E$ is a closed equivalence relation on a Polish space $X$. We must find Borel set $B_{i} \subseteq X$ for $i<\omega$ such that $x E y$ iff for all $i<\omega, x \in B_{i} \Leftrightarrow y \in B_{i}$. Since $X^{2} \backslash E$ is open, it equals $\bigcup_{i<\omega} U_{i} \times V_{i}$ for $U_{i}, V_{i} \subseteq X$ open. Let $U_{i}^{E}=\left\{x \in X \mid \exists y\left(y \in U_{i} \& x E y\right)\right\}$ be the $E$-closure of $U_{i}$ and $V_{i}^{E}$ be the $E$-closure of $V_{i}$. These are analytic sets. Since $U_{i}^{E} \cap V_{i}^{E}=\emptyset$, by Lusin's separation theorem, there are Borel sets $U_{i}^{0}$ such that $U_{i}^{0} \supseteq U_{i}^{E}, U_{i}^{0} \cap V_{i}^{E}=\emptyset$. Recursively we construct Borel sets $U_{i}^{j}$ for $j<\omega$ such that $U_{i}^{j}$ contains the $E$-closure of $U_{i}^{j-1}$ and is disjoint from $V_{i}^{E}$. Let $B_{i}=\bigcup_{j<\omega} U_{i}^{j}$.

Example 1.29. Let $\mathbb{E}_{0}$ be the following equivalence relation on the Cantor space $2^{\omega}:(\eta, \nu) \in \mathbb{E}_{0}$ iff there exists some $n<\omega$ such that for all $m>n, \eta(m)=\nu(m)$.

Proposition 1.30. The relation $\mathbb{E}_{0}$ is non-smooth.

Proof. Recall that all Borel subsets $B$ of a Polish space $X$ have the Baire property: there is an open set $O \subseteq X$ such that $O \Delta B$ is meager. Suppose $\left\{B_{i} \mid i \in \omega\right\}$ are Borel separating sets of $\mathbb{E}_{0}$, so all of them have the Baire property.

Fix some $i<\omega$, and suppose $B_{i}$ is not meager. Then there is some $n<\omega$ and some $s \in 2^{n}$ such that, letting $O_{s}=\left\{\eta \in 2^{\omega} \mid s \triangleleft \eta\right\}, O_{s} \backslash B_{i}$ is meager. Let $t \in 2^{n}$. Since $B_{i}$ is closed under $\mathbb{E}_{0}$, and there is a homeomorphism of $2^{\omega}$ taking $O_{s}$ to $O_{t}$ fixing all $\mathbb{E}_{0}$-classes, $O_{t} \backslash B_{i}$ is also meager. But then $2^{\omega} \backslash B_{i}=\bigcup_{s \in 2^{n}} O_{s} \backslash B_{i}$ is meager, so $B_{i}$ is comeager. This shows that $B_{i}$ is either meager or comeager.

But then,

$$
B=\bigcap\left\{B_{i} \mid i<\omega, B_{i} \text { is comeager }\right\} \cap \bigcap\left\{\sim B_{i} \mid i<\omega, B_{i} \text { is meager }\right\}
$$

is a comeager $\mathbb{E}_{0}$-class, which is a contradiction (since it is countable).

In addition, we have the following dichotomy:

Fact 1.31. HKL90] (Harrington-Kechris-Louveau dichotomy) For every Borel equivalence relation $E$ either $E \leq_{B} \Delta\left(2^{\omega}\right)$ (i.e., $E$ is smooth) or $\mathbb{E}_{0} \sqsubseteq_{c} E$. It follows that $\mathbb{E}_{0}$ is the successor of $\Delta\left(2^{\omega}\right)$

We also mention: 
Corollary 1.32. Suppose $Y$ is a Polish space, and $E$ is a Borel equivalence relation on $Y$ such that all its classes are $G_{\delta}$-subsets. Then $E$ is smooth.

Proof. Suppose $E$ is not smooth. By Fact 1.31, there is a continuous map $f: 2^{\omega} \rightarrow Y$ that reduces $\mathbb{E}_{0}$ to $E$. But then it follows that all the $\mathbb{E}_{0}$-classes are continuous pre-images of $G_{\delta}$ sets, so they are themselves $G_{\delta}$. As they are also dense, this is a contradiction.

1.3. Preliminaries on Choquet spaces. As we mentioned above, when the language is not necessarily countable we will work with Choquet spaces instead of Polish spaces.

Definition 1.33. The Choquet game on a topological space $X$ is a two player game in $\omega$-many rounds. In round $n$, player A chooses a non-empty open set $U_{n} \subseteq V_{n-1}$ (where $V_{-1}=X$ ), and player B responds by choosing a non-empty open subset $V_{n} \subseteq U_{n}$. Player B wins if the intersection $\bigcap\left\{V_{n} \mid n<\omega\right\}$ is not empty.

The strong Choquet game is similar: in round $n$ player A chooses an open set $U_{n} \subseteq V_{n-1}$ and $x_{n} \in U_{n}$, and player B responds by choosing an open set $V_{n} \subseteq U_{n}$ containing $x_{n}$. Again, player B wins when the intersection $\bigcap\left\{V_{n} \mid n<\omega\right\}$ is not empty.

A topological space $X$ is a (strong) Choquet space if player B has a winning strategy in every (strong) Choquet game.

Given a subset $A$ of $X$, we say that $X$ is strong Choquet over $A$ to mean that the points that player A chooses are taken from $A$.

It is easy to see that:

Example 1.34. Every Polish space is strong Choquet.

But for our purposes, we shall need the following example:

Example 1.35. If $X$ is compact (not necessarily Hausdorff) and has a basis consisting of clopen sets then it is strong Choquet.

Proof. In round $n$, player B will choose a clopen set $x_{n} \in V_{n} \subseteq U_{n}$. By compactness, the intersection $\bigcap\left\{V_{n} \mid n<\omega\right\}$ is not empty.

Proposition 1.36. If $X$ is strong Choquet and $\emptyset \neq U \subseteq X$ is $G_{\delta}$, then $U$ is also strong Choquet.

Proof. Suppose $U=\bigcap\left\{W_{n} \mid n<\omega\right\}$ where $W_{n} \subseteq X$ are open. Let $S t$ be a strategy for the strong Choquet game in $X$ and we will describe a strategy $S t_{U}$ for the strong Choquet game in $U$. So we play a game $\partial_{U}$ in $U$, and we run a parallel game $\partial_{X}$ in $X$ as follows. Assume we have already played all the rounds up to $n$ : the sets $U_{i}, V_{i}$ were chosen for $i<n$ in the game $\partial_{U}$, and $U_{i}^{\prime}, V_{i}^{\prime}$ are the corresponding moves in the $\partial_{X}$. The construction will ensure that for all $i<n$, we have 
$U_{i}^{\prime} \cap U=U_{i}, V_{i}^{\prime} \cap U=V_{i}$ and $U_{n}^{\prime} \subseteq W_{n}$. Assume that A plays $\left(U_{n}, x_{n}\right)$, with $x_{n} \in U_{n}$. Pick an open subset $U_{*}$ of $X$ such that $U_{*} \cap U=U_{n}$. We set A's move in the parallel game to be $\left(U_{*} \cap W_{n} \cap V_{n-1}^{\prime}, x_{n}\right)$. Let $V_{n}^{\prime}$ be B's move according to the strategy $S t$. Then in $\partial_{U}$, have B play $V_{n}^{\prime} \cap U$. Note that this set is non-empty since it contains $x_{n}$. This defines a winning strategy for $B$.

\subsection{Context.}

1.4.1. Countable language. In KPS12, the authors gave a natural way of considering $\equiv_{L}^{\alpha}$ and $\equiv_{K P}^{\alpha}$ for a countable complete first order theory $T$ and a countable $\alpha$ as Borel equivalence relations on the space of types $S_{\alpha}(M)$ over a countable model $M$ (this is a Polish space - see Remark 1.3 about the topology). Fix some countable $T$ and $\alpha$.

Definition 1.37. Let $M$ be a countable model. For $p, q \in S_{\alpha}(M)$, we write $p \equiv_{L}^{\alpha, M} q$ iff $\exists a \models p, b \models q\left(a \equiv_{L}^{\alpha} b\right)$ and similarly we define $\equiv_{K P}^{\alpha, M}$.

It will be useful to define the Lascar metric on types:

Definition 1.38. For $p, q \in S_{\alpha}(M)$ let $d_{\alpha}(p, q)=\min \left\{n \in \mathbb{N} \mid \exists a \models p, b \models q\left(d_{\alpha}(a, b) \leq n\right)\right\}$.

Note that:

Remark 1.39. [KPS12, Remark 2.2] Let $M$ be a countable model. By Remark 1.14 for $p, q \in$ $S_{\alpha}(M), p \equiv_{L}^{\alpha, M} q$ iff $\forall a \models p, b \models q\left(a \equiv_{L}^{\alpha} b\right)$ and similarly for $\equiv_{K P}^{\alpha, M}$.

Let $q_{\alpha, M}: S_{\alpha \cdot 2}(M) \rightarrow S_{\alpha}(M)$ be defined by $p(x, y) \mapsto(p\lceil x, q\lceil y)$. This is a continuous map, and hence it is closed. Using this notation, $\equiv_{K P}^{\alpha, M}=q_{\alpha, M} \circ r_{\alpha \cdot 2, M}\left(\equiv_{K P}^{\alpha}\right)$ (see Remark 1.8), and hence $\equiv_{K P}^{\alpha, M}$ is closed. Similarly, the set

$$
F_{n}=\left\{(p, q) \in S_{\alpha}(M) \mid d_{\alpha}(p, q) \leq n\right\}
$$

is closed, and $\equiv_{L}^{\alpha, M}$ is the union $\bigcup_{n<\omega} F_{n}$ hence it is $K_{\sigma}$.

They proved that as far as Borel cardinality goes, this does not depend on the model $M$, even when restricting to a KP strong type:

Fact 1.40. [KPS12, Propositions 2.3, 2.6] Let $M$ and $N$ be any countable models. Then,

(1) $\equiv_{L}^{\alpha, M} \sim_{B} \equiv_{L}^{\alpha, N}$.

(2) For any $a \in \mathfrak{C}, \equiv_{L}^{\alpha, M} \uparrow[\operatorname{tp}(a / M)]_{\equiv_{K P}^{\alpha, M}} \sim_{B} \equiv_{L}^{\alpha, N} \uparrow[\operatorname{tp}(a / N)]_{\equiv_{K P}^{\alpha, N}}$.

One can extend this observation to deal also with pseudo $G_{\delta}$ sets. Suppose $Y \subseteq \mathfrak{C}^{\alpha}$ is a pseudo $G_{\delta}$ set. For a countable model $M, Y_{M}=r_{\alpha, M}(Y) \subseteq S_{\alpha}(M)$ is not necessarily $G_{\delta}$. But in case $Y$ is closed under $\equiv_{L}^{\alpha}$, it is. Indeed, $\mathfrak{C}^{\alpha} \backslash Y$ is pseudo $F_{\sigma}$, and so $r_{\alpha, M}\left(\mathfrak{C}^{\alpha} \backslash Y\right)$ is $F_{\sigma}$. But by Remark 1.14. $r_{\alpha, M}\left(\mathfrak{C}^{\alpha} \backslash Y\right) \cap Y_{M}=\emptyset$. 
For a countable model $M, Y_{M}$ is a Polish space (as every $G_{\delta}$ set is). In addition, changing the model does not change the Borel cardinality:

Proposition 1.41. Fix a pseudo $G_{\delta}$ set $Y \subseteq \mathfrak{C}^{\alpha}$, closed under $\equiv_{L}^{\alpha}$. Then

$$
\equiv_{L}^{\alpha, M} \uparrow Y_{M} \sim_{B} \equiv_{L}^{\alpha, N} \uparrow Y_{N}
$$

Proof. The proof is exactly the same as in [KPS12, Propositions 2.3, 2.6], but we repeat it for completeness. It is enough to establish this when $M \subseteq N$. Let $\pi: S_{\alpha}(N) \rightarrow S_{\alpha}(M)$ be the restriction map. Then $\pi$ is a continuous map that reduces $\equiv_{L}^{\alpha, N}$ to $\equiv_{L}^{\alpha, M}$. By KPS12, Fact 1.7 (i)] there is a Borel section, i.e., a Borel function $\pi^{\prime}: S_{\alpha}(M) \rightarrow S_{\alpha}(N)$ such that $\pi \circ \pi^{\prime}=\mathrm{id}$. Now it follows that $\pi$ and $\pi^{\prime}$ restricted to $Y_{M}$ and $Y_{N}$ witness Borel bi-reducibility.

This allows us to refer to the Borel cardinality of $\equiv_{L}^{\alpha} Y Y$ without specifying the model.

1.4.2. Countable or uncountable language. Let $T$ be any complete first order theory and $\alpha$ any ordinal. In order to state our theorem in full generality, we shall need the following definition:

Definition 1.42. We say that a set $Y \subseteq \mathfrak{C}^{\alpha}$ for some small $\alpha$ is pseudo strong Choquet if $Y_{M}$ is strong Choquet for all $M$.

Example 1.43. Pseudo closed and pseudo $G_{\delta}$ sets which are closed under $\equiv_{L}^{\alpha}$ are pseudo strong Choquet by the observation after Fact 1.40 and Proposition 1.36.

Remark 1.44. For countable $T$ and $\alpha$, "pseudo strong Choquet" is the correct analog of pseudo $G_{\delta}$ for sets closed under $\equiv_{L}^{\alpha}$. By [Kec95, Theorem 8.17] if $Y \subseteq \mathfrak{C}^{\alpha}$ is such a set, then $Y$ is pseudo strong Choquet iff $Y$ is pseudo $G_{\delta}$ iff $Y_{M}$ is Polish for every $M$.

1.5. Results. Our main theorem, proved in Section 4, is:

Main Theorem A. Suppose $T$ is a complete countable first-order theory, $\alpha$ a countable ordinal, and suppose $Y$ is a pseudo $G_{\delta}$ subset of $\mathfrak{C}^{\alpha}$ which is closed under $\equiv_{L}^{\alpha}$. If for some $a \in Y,[a]_{\equiv_{L}^{\alpha}}$ is not d-bounded, then $\equiv_{L}^{\alpha} \uparrow Y$ is non-smooth.

Remark 1.45. This theorem remains true also for many-sorted countable theories, with the obvious adjustments.

We immediately get Conjecture 1 of [KPS12]:

Corollary 1.46. Suppose $T$ and $\alpha$ are as above. Suppose $K \subseteq \mathfrak{C}^{\alpha}$ is a KP strong type. If $\equiv_{L}^{\alpha} \uparrow K$ is not d-bounded, then $\equiv_{L}^{\alpha} \uparrow K$ is non-smooth. In particular, by Remark 1.21, if $\equiv_{L}^{\alpha} \uparrow K$ is not trivial, then it is non-smooth. 
Proof. Observe that if $\equiv_{L}^{\alpha} \uparrow K$ is not $d$-bounded, then there is a $\equiv_{L}^{\alpha}$-class inside $K$ which is not $d$-bounded (else all classes will have the same bound, since they are conjugates).

Corollary 1.47. Suppose $T$ and $\alpha$ are as above. Then $\equiv_{L}^{\alpha}$ is closed iff it is smooth.

Proof. If $\equiv_{L}^{\alpha}$ is not closed, then $\equiv_{L}^{\alpha} \neq \equiv_{K P}^{\alpha}$, so there is a KP strong type $K$ such that $\equiv_{L}^{\alpha} \uparrow K$ is not trivial, so $\equiv_{L}^{\alpha} \uparrow K$ is not smooth, so also $\equiv_{L}^{\alpha}$. The other direction follows from Proposition 1.28 .

Remark 1.48. Since our main result concerns $\mathbb{E}_{0}$, it actually says something about the "definable cardinality" of $\equiv_{L}^{\alpha}$, i.e., it is stronger than just saying something about the Borel cardinality of $\equiv_{L}^{\alpha}$, but also allows reductions to be "definable". In the proof of Proposition 1.30, we showed that there are no separating sets for $\mathbb{E}_{0}$ with the Baire property. In any reasonable interpretation of the term, any "definable" reduction of $\mathbb{E}_{0}$ to $\Delta(Y)$ for some Polish space $Y$ will give rise to such separating sets. So our main result implies that the "definable cardinality" of $\equiv_{L}^{\alpha}$ is greater than $\Delta\left(2^{\omega}\right)$. We will not give an exact definition of "definable cardinality" (see more in [BK96, Chapter 8]).

For a general language and $\alpha$ we have:

Main Theorem B. [Simplified version] Suppose $T$ is a complete first-order theory, $\alpha$ a small ordinal. Suppose $Y \subseteq \mathfrak{C}^{\alpha}$ is closed under $\equiv_{L}^{\alpha}$ and for some $a \in Y,[a]_{\equiv_{L}^{\alpha}}$ is not d-bounded. Suppose $Y$ is pseudo strong Choquet. Then $\left|Y / \equiv_{L}^{\alpha}\right| \geq 2^{\aleph_{0}}$.

The full theorem says a bit more, see 5.1

Corollary 1.49. Fact 1.1 holds for any theory $T$ and any small ordinal $\alpha$.

Proof. (1), (2) and (3) follow immediately from Main Theorem B. (3) is also connected to Corollary 1.32 .

(4) Suppose $T$ is small. Let $n<\omega$, let $a$ be some tuple of length $n$ and let $Y=S_{n}(a)$. This is a countable Polish space. Thus every subset of $Y$ is $G_{\delta}$, in particular the set

$$
Q=\left\{q \in S_{n}(a) \mid \forall b \models q\left(b \equiv_{L}^{n} a\right)\right\}
$$

(which can also can also be defined with $\exists$ ). Let $M$ be any countable model containing $a$. Then the restriction map $\pi: S(M) \rightarrow S(a)$ is continuous. Thus, $\pi^{-1}(Q)$ is also $G_{\delta}$. But it is exactly the Lascar strong type of $a$ in $S(M)$. By (3), this class is $d$-bounded, but then by Remark 1.21 $\equiv_{L}^{n} \uparrow[a]_{\equiv_{K P}^{n}}$ is trivial and hence $\equiv_{K P}^{n}=\equiv_{L}^{n}$. 


\section{Descriptive Set theoretic lemmas}

2.1. Polish spaces. Given a group $\Gamma$ of homeomorphisms of a topological space $X$, we use $E_{\Gamma}^{X}$ to denote the corresponding orbit equivalence relation. Although the following fact can be seen as a consequence of the proof of [BK96, Theorem 3.4.5], for the sake of completeness we provide a proof.

Theorem 2.1. Suppose that $X$ is a perfect Polish space, $\Gamma$ is a group of homeomorphisms of $X$ with a dense orbit, and $R \subseteq X \times X$ is a meager set. Then there is a continuous, injective homomorphism $\phi: 2^{\omega} \rightarrow X$ from $\left(\mathbb{E}_{0}, \sim \mathbb{E}_{0}\right)$ to $\left(E_{\Gamma}^{X}, \sim R\right)$.

Proof. We use $1_{\Gamma}$ to denote the identity element of $\Gamma$. Given a natural number $n$ and a sequence $\left\langle\gamma_{i} \mid i<n\right\rangle$ of elements of $\Gamma$, we use $\prod_{i<n} \gamma_{i}$ to denote $1_{\Gamma}$ when $n=0$, and the product $\gamma_{0} \cdots \gamma_{n-1}$ when $n>0$. When $\left\langle\gamma_{i} \mid i<n\right\rangle$ is constant with value $\gamma$, we also use $\gamma^{n}$ to denote $\prod_{i<n} \gamma_{i}$.

As $X$ is perfect, the set of pairs of distinct points of $X$ is comeager, so there is a decreasing sequence $\left\langle U_{n} \mid n \in \mathbb{N}\right\rangle$ of dense, irreflexive, open, symmetric subsets of $X \times X$ whose intersection is disjoint from $R$. We will recursively construct group elements $\gamma_{n} \in \Gamma$, with which we associate the products $\gamma_{s}=\prod_{i<n} \gamma_{i}^{s(i)}$, for all $n \in \mathbb{N}$ and $s \in 2^{n}$. We will simultaneously construct points $x_{n} \in X$ and open neighborhoods $X_{n}$ of $x_{n}$ with the following properties:

(1) $\overline{X_{n+1}} \subseteq X_{n} \cap\left(\gamma_{n}^{-1} \cdot X_{n}\right)$.

(2) $\forall s \in 2^{n+1} \operatorname{diam}\left(\gamma_{s} \cdot X_{n+1}\right) \leq 1 / n$.

(3) $\forall s, t \in 2^{n+1}\left(s(n) \neq t(n) \Rightarrow\left(\gamma_{s} \cdot X_{n+1}\right) \times\left(\gamma_{t} \cdot X_{n+1}\right) \subseteq U_{n}\right)$.

We begin by fixing an arbitrary point $x_{0} \in X$ and setting $X_{0}=X$.

Suppose now that $n \in \mathbb{N}$ and we have already found $\left\langle\gamma_{m} \mid m<n\right\rangle, x_{n}$, and $X_{n}$. The fact that $\Gamma$ consists of homeomorphisms then ensures that the set

$$
V_{n}=\bigcap\left\{\left(\gamma_{s} \times \gamma_{t}\right)^{-1}\left(U_{n}\right) \mid(s, t) \in 2^{n} \times 2^{n}\right\}
$$

is dense and open, so the fact that $\Gamma$ has a dense orbit yields $\gamma_{n} \in \Gamma$ and $x_{n+1} \in X_{n} \cap\left(\gamma_{n}^{-1} \cdot X_{n}\right)$ for which $\left(x_{n+1}, \gamma_{n} \cdot x_{n+1}\right) \in V_{n}$. As $\Gamma$ consists of homeomorphisms and $U_{n}$ is symmetric, there is an open neighborhood $X_{n+1}$ of $x_{n+1}$ satisfying conditions (1) - (3). This completes the recursive construction.

Conditions (1) and (2) ensure that we obtain a continuous function $\phi: 2^{\omega} \rightarrow X$ by setting $\phi(c)=\lim _{n \rightarrow \infty} \gamma_{c \mid n} \cdot x_{n}$. To see that $\phi$ is a homomorphism from $\mathbb{E}_{0}$ to $E_{\Gamma}^{X}$, it is sufficient to observe that if $k \in \mathbb{N}, s \in 2^{k}$, and $y \in 2^{\omega}$, then

$$
\phi(s \frown y)=\lim _{n \rightarrow \infty} \gamma_{s \frown y \nmid n} \cdot x_{n}=\lim _{n \rightarrow \infty} \gamma_{s} \gamma_{(0)^{k} \frown y \uparrow n} \cdot x_{n}=\gamma_{s} \cdot \phi\left((0)^{k} \frown y\right) .
$$


Observe now that if $y, z \in 2^{\omega}$ and $y(n) \neq z(n)$, then conditions (1) and (3) ensure that $(\phi(y), \phi(z)) \in\left(\gamma_{y \uparrow(n+1)} \cdot X_{n+1}\right) \times\left(\gamma_{z \uparrow(n+1)} \cdot X_{n+1}\right) \subseteq U_{n}$, so the irreflexivity of $U_{n}$ yields the injectivity of $\phi$, and the fact that $\left\langle U_{n} \mid n \in \mathbb{N}\right\rangle$ is a decreasing sequence whose intersection is disjoint from $R$ ensures that $\phi$ is a homomorphism from $\sim \mathbb{E}_{0}$ to $\sim R$.

Given $R \subseteq X \times X$ and $x \in X$, define $R_{x}=\{y \in X \mid x R y\}$.

Theorem 2.2. Suppose that $X$ is a Polish space, $\left\langle R_{n} \mid n \in \mathbb{N}\right\rangle$ is a sequence of $F_{\sigma}$ subsets of $X \times X, \Gamma$ is a group of homeomorphisms of $X$, and $\mathcal{O} \subseteq X$ is an orbit of $\Gamma$ with the property that for all $n \in \mathbb{N}$ and open sets $U \subseteq X$ intersecting $\mathcal{O}$, there are distinct $x, y \in \mathcal{O} \cap U$ with $\mathcal{O} \cap\left(R_{n}\right)_{x} \cap\left(R_{n}\right)_{y}=\emptyset$. Then there is a continuous, injective homomorphism $\phi: 2^{\omega} \rightarrow \overline{\mathcal{O}}$ from $\left(\mathbb{E}_{0}, \sim \mathbb{E}_{0}\right)$ to $\left(E_{\Gamma}^{X}, \sim \bigcup\left\{R_{n} \mid n \in \mathbb{N}\right\}\right)$.

Proof. In light of Theorem 2.1 it is sufficient to show that $\overline{\mathcal{O}}$ is perfect and $\bigcup\left\{R_{n} \mid n \in \mathbb{N}\right\} \uparrow \overline{\mathcal{O}}$ is meager. For the former, observe that if $U \subseteq X$ is an open set intersecting $\overline{\mathcal{O}}$, then it intersects $\mathcal{O}$, so there are distinct $x, y \in \mathcal{O} \cap U$. For the latter, it is sufficient to check that each of the sets $\sim R_{n} \uparrow \overline{\mathcal{O}}$ is dense. Towards this end, suppose that $U, V \subseteq X$ are open sets intersecting $\overline{\mathcal{O}}$, and therefore $\mathcal{O}$. Then there exist $x, y \in \mathcal{O} \cap U$ with $\mathcal{O} \cap\left(R_{n}\right)_{x} \cap\left(R_{n}\right)_{y}=\emptyset$, as well as $z \in \mathcal{O} \cap V$, so $\neg x R_{n} z$ or $\neg y R_{n} z$, thus $\sim R_{n} \cap \mathcal{O} \cap(U \times V) \neq \emptyset$.

We are going to apply this in our context via:

Corollary 2.3. Let $T$ be a countable first order theory, let $\alpha$ be a countable ordinal and $M a$ countable model. Let $Y$ be a Polish subspace of $S_{\alpha}(M)$ that is closed under $\equiv_{L}^{\alpha, M}$. Suppose that there is some $\mathbf{x} \in Y$ such that for every open set $U \ni \mathbf{x}$ and for all $N \in \mathbb{N}$, there exist some $\sigma \in \operatorname{Aut} f_{L}(\mathfrak{C})$ such that:

(1) The automorphism $\sigma^{*}$ that $\sigma$ induces on $S_{\alpha}(M)$ fixes $Y$ setwise.

(2) $\sigma^{*}(\mathbf{x}) \in U$ and $N<d_{\alpha}\left(\sigma^{*}(\mathbf{x}), \mathbf{x}\right)$ (see Definition $\mathbf{1 . 3 8}$ ).

Then there is a continuous, injective homomorphism $\phi: 2^{\omega} \rightarrow Y$ from $\left(\mathbb{E}_{0}, \sim \mathbb{E}_{0}\right)$ to $\left(\equiv_{L}^{\alpha, M}, \sim \equiv_{L}^{\alpha, M}\right)$. In particular, $\equiv_{L}^{\alpha, M} \uparrow Y$ is not smooth.

Proof. For $n<\omega$, let $R_{n}$ be the closed set $\left\{(p, q) \in Y \times Y \mid d_{\alpha}(p, q) \leq n\right\}$. Let $\Gamma$ be the group of homeomorphisms of $Y$ which are induced by automorphisms in Aut $f_{L}(\mathfrak{C})$ which fix $Y$ setwise. Let $\mathcal{O}$ be the orbit of $\mathbf{x}$ under $\Gamma$.

Let $n \in \mathbb{N}$ and let $W$ be an open set which intersects $\mathcal{O}$. Then for some $\gamma \in \Gamma, \gamma(\mathbf{x}) \in W$. Let $U=\gamma^{-1}(W)$. Then for some $\sigma \in \operatorname{Aut} f_{L}(\mathfrak{C}), \sigma^{*} \in \Gamma, \sigma^{*}(\mathbf{x}) \in U$ and $2 n<d_{\alpha}\left(\sigma^{*}(\mathbf{x}), \mathbf{x}\right)$. Let $x=\gamma(\mathbf{x})$ and $y=\gamma \sigma^{*}(\mathbf{x})$. So $d_{\alpha}(x, y)=d_{\alpha}\left(\mathbf{x}, \sigma^{*}(\mathbf{x})\right)>2 n$ and so $x$ and $y$ are distinct and $\mathcal{O} \cap\left(R_{n}\right)_{x} \cap\left(R_{n}\right)_{y}=\emptyset$. 
2.2. Choquet spaces. In order to prove Main Theorem $\mathrm{B}$, we have to work over a model $M$ of possibly uncountable size, hence $S(M)$ is no longer a Polish space. The idea is to mimic the proof of Main Theorem $\mathrm{A}$. i.e., construct step-by-step an embedding of $\mathbb{E}_{0}$. In the countable case we use completeness at the limit stage, but here we use the winning strategy in the (strong) Choquet game.

The main observation is that Theorem 2.1 has a natural analog in the Choquet context:

Theorem 2.4. Suppose that $X$ is regular topological space, $\Gamma$ is a group of homeomorphisms of $X$ and $\mathcal{O}$ an orbit of $\Gamma$ such that $X$ is Choquet over $\mathcal{O}$. Suppose that for $n<\omega, V_{n} \subseteq X \times X$ is a $G_{\delta}$ subset such that $V_{n} \uparrow \overline{\mathcal{O}} \times \overline{\mathcal{O}}$ is dense. Then there is a map $\phi: 2^{\omega} \rightarrow \mathcal{P}(X)$ such that for every $y, z \in 2^{\omega}:$

- $\phi(y)$ is a non-empty closed $G_{\delta}$ subset of $X$.

- If $z \mathbb{E}_{0} y$ then there is some $\gamma \in \Gamma$ such that $\gamma \cdot \phi(z)=\phi(y)$.

- If $\sim z \mathbb{E}_{0} y$ then $(\phi(y) \times \phi(z)) \subseteq \bigcap_{n<\omega} V_{n}$.

Proof. The proof follows along the lines of the proof of Theorem 2.1. The main difference is that in condition (2) in the construction, instead of controlling the diameter of the open sets, one has to refine them so that they obey the winning strategy of player B in the suitable Choquet game over $\mathcal{O}$.

From this we get the following analog of 2.2

Theorem 2.5. Suppose that $X$ is a topological space, $\left\langle R_{n} \mid n \in \mathbb{N}\right\rangle$ is a sequence of $F_{\sigma}$ subsets of $X \times X, \Gamma$ is a group of homeomorphisms of $X$, and $\mathcal{O} \subseteq X$ is an orbit of $\Gamma$ with the property that for all $n \in \mathbb{N}$ and open sets $U \subseteq X$ intersecting $\mathcal{O}$, there are distinct $x, y \in \mathcal{O} \cap U$ with $\mathcal{O} \cap\left(R_{n}\right)_{x} \cap\left(R_{n}\right)_{y}=\emptyset$. If $X$ is strong Choquet over $\mathcal{O}$ then the conclusion of Theorem 2.4 holds with $V_{n}=\sim R_{n}$.

And:

Corollary 2.6. Let $T$ be any first order theory with language $L$, let $\alpha$ be any ordinal and $M a$ model. Let $Y$ be a subspace of $S_{\alpha}(M)$ that is closed under $\equiv_{L}^{\alpha, M}$. Suppose that there is

(1) Some $\mathbf{x} \in Y$.

(2) A countable sub-language $L^{\prime}$ of $L$, a countable set $M^{\prime} \prec M \uparrow L^{\prime}$ and a countable sub-tuple of the first $\alpha$ variables which for simplicity we will assume to be the initial segment of length $\beta$.

(3) A countable subgroup $\Sigma \leq$ Aut $f_{L}(\mathfrak{C})$ of automorphisms that fix $M^{\prime}$ and $M$ setwise.

Such that: 
(1) With the topology induced on $Y$ by $L^{\prime}, M^{\prime}$, and $\beta$ (the one generated by formulas in $\left.L_{\beta}^{\prime}\left(M^{\prime}\right)\right), Y$ is strong Choquet over $\Sigma \cdot \mathbf{x}$.

(2) For every open set $U \ni \mathbf{x}$ in the induced topology and for all $N \in \mathbb{N}$, there exist some $\sigma \in \Sigma$ such that $\sigma^{*}(\mathbf{x}) \in U$ and, letting $\mathbf{x}^{\prime}=\mathbf{x} \mid L_{\beta}\left(M^{\prime}\right), N<d_{\beta}^{\prime}\left(\sigma^{*}\left(\mathbf{x}^{\prime}\right), \mathbf{x}^{\prime}\right)\left(d_{\beta}^{\prime}\right.$ is the Lascar metric of the language $L^{\prime}$ ).

Then there is a map $\phi: 2^{\omega} \rightarrow \mathcal{P}(Y)$ such that for every $y, z \in 2^{\omega}$ :

- $\phi(y)$ is a non-empty closed $G_{\delta}$ subset of $Y$.

- If $z \mathbb{E}_{0} y$ then there is a some $\gamma \in \Gamma$ such that $\gamma \cdot \phi(z)=\phi(y)$.

- If $\sim z \mathbb{E}_{0} y$ then $(\phi(y) \times \phi(z)) \cap \equiv_{L}^{\alpha, M}=\emptyset$.

Proof. Similar to 2.3. Note that if $p, q \in S_{\alpha}(M)$ and $p \nmid L_{\beta}^{\prime}\left(M^{\prime}\right), q \uparrow L_{\beta}^{\prime}\left(M^{\prime}\right)$ are not $\equiv_{L}^{\beta, M^{\prime}}$ equivalent, then $p, q$ are not $\equiv_{L}^{\alpha, M}$-equivalent.

The following lemma will not be used directly, but its proof will give insight into the proof of Main theorem B

Lemma 2.7. Suppose $(X, \tau)$ is a Choquet space with topology $\tau$. Let $B \subseteq \mathcal{P}(X)$ be a base for $\tau$, and assume it is closed under finite intersections. Let $B_{0} \subseteq B$. Then there exists $B_{0} \subseteq B_{1} \subseteq B$ such that $\left|B_{1}\right| \leq\left|B_{0}\right|+\aleph_{0}$ and $\left(X, \tau_{B_{1}}\right)$ is Choquet, where $\tau_{B_{1}}$ is the topology generated by $B_{1}$.

Proof. Let $S t$ be a winning strategy for player B in the Choquet game of $(X, \tau)$. Let $s=\left\langle U_{i} \mid i \leq n\right\rangle$ be a finite sequence of elements of $B_{0}$. Suppose $s$ consists of a legal $n+1$-play of player A, where player B plays his moves according to $S t$ for $i<n$. Let $V_{s}$ be a nonempty basic open set contained in player B's play in the $n$ 'th round of his move according to $S t$. Let $B_{0}^{1}$ be the closure under finite intersections of $B_{0} \cup\left\{V_{s} \mid s \in B_{0}^{<\omega}\right\}$. This is a subset of $B$. Repeat this recursively to construct $B_{0}^{n}$ for $n<\omega$, and let $B_{1}=\bigcup_{n<\omega} B_{0}^{n}$. Then $B_{1}$ satisfies the cardinality demand. Let us see that $\left(X, \tau_{B_{1}}\right)$ is Choquet. For this we must describe a winning strategy for player B.

So suppose $\left\langle\left(U_{i}, V_{i}\right) \mid i<n\right\rangle$ is a legal play of the Choquet game in $\tau_{B_{1}}$ (where $U_{i}$ is played by player $\mathrm{A}$ and $V_{i}$ is played by player B), and player A chooses $U_{n}$. Suppose that:

- There are basic open sets $U_{i}^{\prime} \in B_{1}$ and open sets $V_{i}^{\prime} \in \tau$ for $i<n$ such that $\left\langle\left(U_{i}^{\prime}, V_{i}^{\prime}\right) \mid i<n\right\rangle$ is a play of the Choquet game compatible with $S t, U_{i}^{\prime} \subseteq U_{i}$ and $V_{i} \subseteq V_{i}^{\prime}$.

Let $U_{n}^{\prime} \in B_{1}$ be such that $U_{n}^{\prime} \subseteq U_{n}$. There is some $m<\omega$ such that $U_{i}^{\prime} \subseteq B_{0}^{m}$ for all $i<n$ and let $s=\left\langle U_{i}^{\prime} \mid i \leq n\right\rangle$. By construction of $B_{0}^{m+1}, V_{s} \in B_{1}$ so let player B play $V_{s}$.

If this does not hold, let player B play any set.

Now it is easy to see that if player B plays according to this strategy, then he will win the game. 


\section{The small CASE}

Here we prove Main Theorem $\mathrm{A}$ under the assumption that a consequence of smallness holds, namely that the conclusion of Fact 1.1 (4) holds. This result is superseded by Theorem 4.12 in the next section, and the reader may skip it if desired.

Assume that $T$ is a complete theory in a countable language $L$ and that $\mathfrak{C}$ is a monster model for $T$.

Claim 3.1. Suppose that $A$ is a countable set and that $\left\{\sigma_{i} \mid i<\omega\right\}$ is a set of automorphisms of $\mathfrak{C}$. Then there is a countable model $N \supseteq A$ such that $\sigma_{i} \uparrow N$ is an automorphism of $N$ for all $i<\omega$.

Proof. Let $M_{0}$ be some model containing $A$, and for $n>0$, let $M_{n}$ be a countable model containing $\bigcup_{j \in \mathbb{Z}, i<\omega} \sigma_{i}^{(j)}\left(M_{n-1}\right)$. Let $N=\bigcup_{n<\omega} M_{n}$.

Definition 3.2. Call a countable model $M$ of $T$ nice if the following conditions hold:

(1) For every pair of finite tuples $a, b \in M^{k}$, if $a \equiv_{L}^{k} b$ then there is a Lascar strong automorphism $\sigma$ of $M$ (i.e., $\sigma \in$ Aut $f_{L}(\mathfrak{C}) \cap$ Aut $(M)$ ) that maps $a$ to $b$. Moreover, $\sigma$ has minimal bound (see Fact 1.17 (2)) among all automorphisms in Aut $f_{L}(\mathfrak{C})$ that map $a$ to $b$.

(2) For every finite tuple $a \in M^{k}$, and every $n<\omega$, if there are $c_{1}, c_{2} \in \mathfrak{C}^{k}$ such that $c_{1} \equiv_{L}^{k} a \equiv_{L}^{k} c_{2}$ and $d_{k}\left(c_{1}, c_{2}\right)>n$, then there are such $c_{1}, c_{2}$ in $M^{k}$.

(3) For all finite tuples $a, b \in M^{k}$ and $a^{\prime} \in M^{k^{\prime}}$ and every $n<\omega$, if there is some $b^{\prime}$ such that $d_{k+k^{\prime}}\left(a \frown a^{\prime}, b \frown b^{\prime}\right) \leq n$, then there is some such $b^{\prime} \in M^{k^{\prime}}$.

Lemma 3.3. Nice models exist. Moreover, for every countable set $A$, there is a nice model $M$ that contains it.

Proof. Let $M_{0}$ be any countable model containing $A$. Recursively choose $M_{n+1}$ to satisfy (1)-(3) relative to $M_{n}$ (using Claim 3.1) and set $M=\bigcup_{n<\omega} M_{n}$.

Fix a countable ordinal $\alpha$ and a pseudo $G_{\delta}$ set $Y$. Assume that:

Assumption A. (1) $\alpha$ is infinite.

(2) The Lascar strong type of every finite sub-tuple of a tuple from $Y$ is $d$-bounded.

Remark 3.4. By Fact 1.1 (4), if $T$ is small, then for finite tuples, $\equiv_{K P}=\equiv_{L}$, so this assumption is satisfied when $\alpha$ is infinite if $T$ is small, and Corollary 1.46 is trivial for finite $\alpha$ (given Fact 1.1 $(1))$.

Theorem 3.5. Main Theorem $\not$ holds under Assumption $A$,

Namely, suppose $\alpha$ and $Y$ are as above and for some $\bar{a} \in Y,[\bar{a}]_{\equiv_{L}^{\alpha}}$ is not not d-bounded. Then $\equiv_{L}^{\alpha} \uparrow Y$ is non-smooth. 
Proof. Choose a nice model $M$ (by Lemma 3.3 ) that contains $\bar{a}$.

Let $\mathbf{x}=p=\operatorname{tp}(\bar{a} / M)$. We shall show that corollary 2.3 applies with $Y$ there being $Y_{M}$ (see Proposition 1.41).

Suppose $U$ is some open set containing $p$, and $N$ is some number. In general, $U$ has the form $[\varphi]$ for some $\varphi \in L_{\alpha}(M)$. But in our case, since $M \models \varphi(\bar{a})$, we may replace $U$ with a smaller open neighborhood of $p$ defined by a formula of the form $x=c$, where $x$ is the tuple of first $k$ variables and $c=\bar{a} \uparrow k$.

Let $B$ be a bound on the diameter of $[c]_{\equiv_{L}^{k}}$. Since the class of $\bar{a}$ is not of bounded diameter, by compactness there must be some finite extension of $c$ to a longer sub-tuple $c \frown c^{\prime}=\bar{a} \uparrow\left(k+k^{\prime}\right)$ such that the $\equiv_{L}^{k+k^{\prime}}$-class of $c \frown c^{\prime}$ has diameter greater than $2 N+4 B$.

There are two tuples $f_{1} \frown f_{1}^{\prime}$ and $f_{2} \frown f_{2}^{\prime}$ in $\mathfrak{C}$ and $\left[c \frown c^{\prime}\right]_{\equiv_{L}^{k+k^{\prime}}}$ such that $d_{k+k^{\prime}}\left(f_{1} \frown f_{1}^{\prime}, f_{2} \frown f_{2}^{\prime}\right)>$ $2 N+2 B$. Since $M$ is nice, we may assume that these tuples are in $M$.

By choice of $B$, niceness of $M$ and Remark 1.18, there are $c^{\prime \prime}$ and $c^{\prime \prime \prime}$ in $M$ such that

$$
d_{k+k^{\prime}}\left(f_{1} \frown f_{1}^{\prime}, c \frown c^{\prime \prime}\right), d_{k+k^{\prime}}\left(f_{2} \frown f_{2}^{\prime}, c \frown c^{\prime \prime \prime}\right) \leq 2 B
$$

So $d_{k+k^{\prime}}\left(c \frown c^{\prime \prime}, c \frown c^{\prime \prime \prime}\right)>2 N$. It follows that for one of $c^{\prime \prime}, c^{\prime \prime \prime}$, say $c^{\prime \prime}, d_{k+k^{\prime}}\left(c \frown c^{\prime}, c \frown c^{\prime \prime}\right)>N$ (but $c \frown c^{\prime} \equiv{ }_{L}^{k+k^{\prime}} c \frown c^{\prime \prime}$ ).

Let $\sigma$ be a Lascar strong automorphism of $M$ that maps $c \frown c^{\prime}$ to $c \frown c^{\prime \prime}$. Since $\sigma$ fixes $c$, $q=\sigma^{*}(p) \in U$. But $q$ is realized by a tuple that contains $c \frown c^{\prime \prime}$, and hence $d_{\alpha}(q, p)>N$.

\section{The countable case}

Assume that $\alpha$ is a countable ordinal, $T$ is a complete theory in a countable language $L$ and $\mathfrak{C}$ is a monster model for $T$.

Definition 4.1. For a formula $\alpha(x, a)$ over a tuple $a$ and an automorphism $\sigma, \sigma(\alpha)=\alpha(x, \sigma(a))$.

Definition 4.2. Suppose $C \subseteq \mathfrak{C}^{\alpha}$ is an $\equiv_{L}^{\alpha}$-class. A formula $\varphi \in L_{\alpha}(\mathfrak{C})$ is said to be $C$-generic if finitely many translates of it under Aut $f_{L}(\mathfrak{C})$ cover $C$. The formula $\varphi$ is said to be $C$-weakly generic if there is a non- $C$-generic formula $\psi \in L_{\alpha}(\mathfrak{C})$ such that $\varphi \vee \psi$ is $C$-generic. A partial $p \subseteq L_{\alpha}(\mathfrak{C})$ is said to be $C$-generic ( $C$-weakly generic) if all its formulas are.

Claim 4.3. The formulas which are not $C$-weakly generic form an ideal.

Proof. Suppose $\varphi_{1}, \varphi_{2}$ are not $C$-weakly generic and we have to show that $\varphi_{1} \vee \varphi_{2}$ is also not $C$-weakly generic. If not, there is some non- $C$-generic $\psi$ such that $\varphi_{1} \vee \varphi_{2} \vee \psi$ is $C$-generic. But $\varphi_{2} \vee \psi$ is not $C$-generic (since $\varphi_{2}$ is not $C$-weakly generic), so we get a contradiction.

By $\varphi \vdash_{C} \psi$ we mean that for every $a \in C$, if $\mathfrak{C} \models \varphi(a)$ then $\mathfrak{C} \models \psi(a)$. 
Remark 4.4. If $\varphi \vdash_{C} \psi$ and $\sigma \in \operatorname{Aut} f_{L}(\mathfrak{C})$ then $\sigma(\varphi) \vdash_{C} \sigma(\psi)$, so if $\varphi$ is (weakly) generic, then so is $\psi$.

Definition 4.5. Suppose $p$ is a weakly generic (partial) type over $\mathfrak{C}$. Suppose furthermore that $p$ is closed under conjunctions. Say that it is $C$-proper if there is a non- $C$-generic formula $\psi$ such that for all $\varphi \in p, \varphi \vee \psi$ is $C$-generic. In general, $p$ is $C$-proper when its closure under finite conjunctions is.

Fix an $\equiv_{L}^{\alpha}$-class $C$. When we write "(weakly) generic" and "proper", we mean " $C$-(weakly) generic" and " $C$-proper".

Example 4.6. If $p$ is generic, then it is proper.

An easy and well known combinatorial lemma is the following:

Lemma 4.7. If $(P,<)$ is a directed order, $k<\omega$ and $f: P \rightarrow k$ is some function, then there is a cofinal $f$-homogeneous set $P_{0} \subseteq P$ : there is some $i<k$ such that $f^{-1}(i)$ is cofinal.

Proof. Suppose not. So for each $i<k$, the $f^{-1}(i)$ is not cofinal, for some $p_{i} \in P$, for no $q \geq p_{i}$, $f(q)=i$. Let $p$ be $\geq p_{i}$ for every $i<k$. Then $p \geq p_{f(p)}-$ contradiction.

Lemma 4.8. Suppose $p \subseteq L_{\alpha}(\mathfrak{C})$ is a partial proper type as witnessed by $\psi$. Suppose that $\bigvee_{i<n} \varphi_{i} \bigvee$ $\psi^{\prime}$ covers $C$ and that $\psi^{\prime} \vee \psi$ is non-generic. Then for some $i<n, p \cup\left\{\varphi_{i}\right\}$ is proper.

Proof. We may assume that $p$ is closed under conjunctions. For each formula $\zeta \in p$, by assumption we have:

$$
\zeta \vee \psi \vdash_{C} \bigvee_{i<n}\left(\varphi_{i} \wedge \zeta\right) \vee \psi^{\prime} \vee \psi
$$

So by Remark 4.4, the right hand side is generic.

For each $\zeta \in p$ and $k<n$, let $\zeta_{k}=\bigvee_{k \leq i<n}\left(\varphi_{i} \wedge \zeta\right) \vee \psi^{\prime} \vee \psi$. Let $k_{\zeta}<n$ be maximal such that $\zeta_{k}$ is generic (must exist since $\zeta_{0}$ is generic), so $\zeta_{k+1}$ is non-generic. By Lemma 4.7, for some $k<n$, the set $\left\{\zeta \mid k_{\zeta}=k\right\}$ is cofinal in the order $\zeta_{1}>\zeta_{2} \Leftrightarrow \zeta_{1} \vdash \zeta_{2}$. Fix some $\chi \in p$ such that $k_{\chi}=k$. We will show that $p \cup\left\{\varphi_{k}\right\}$ is proper, as witnessed by $\chi_{k+1}$.

Suppose $\zeta \in p$. Let $\zeta^{\prime}=\zeta \wedge \chi$, and $\zeta^{\prime \prime} \vdash \zeta^{\prime}$ be such that $k_{\zeta^{\prime \prime}}=k$. Then $\left(\zeta^{\prime \prime} \wedge \varphi_{k}\right) \vee \zeta_{k+1}^{\prime \prime}$ is generic. Since $\zeta^{\prime \prime} \wedge \varphi_{k} \vdash \zeta \wedge \varphi_{k}$ and $\zeta_{k+1}^{\prime \prime} \vdash \chi_{k+1},\left(\zeta \wedge \varphi_{k}\right) \vee \chi_{k+1}$ is also generic and we are done.

Lemma 4.9. If $p \subseteq L_{\alpha}(\mathfrak{C})$ is a partial proper type, then for every formula $\varphi \in L_{\alpha}(\mathfrak{C})$, either $p \cup\{\varphi\}$ is proper or $p \cup\{\neg \varphi\}$ is proper.

Proof. Apply Lemma 4.8 with $n=2, \varphi_{0}=\varphi, \varphi_{1}=\neg \varphi$ and $\psi^{\prime}=\perp$ (i.e., $\forall x(x \neq x)$ ).

Note that if we do not care about properness but only about weak genericity, then this follows directly from Claim 4.3 . 
Proposition 4.10. Suppose that $p \subseteq L_{\alpha}(\mathfrak{C})$ is a partial proper type, and $\varphi \in p$. Then there are $\sigma_{0}, \ldots \sigma_{n-1} \in$ Aut $f_{L}(\mathfrak{C})$ such that for every $\sigma \in$ Aut $f_{L}(\mathfrak{C})$, there exists some $i<n$ such that $p \cup\left\{\sigma\left(\sigma_{i}(\varphi)\right)\right\}$ is proper.

Proof. Since $p$ is proper, there is some non-generic formula $\psi(x)$ that witnesses it. In particular, there is some $n<\omega$ and some $\sigma_{0}, \ldots, \sigma_{n-1} \in \operatorname{Aut} f_{L}(\mathfrak{C})$ such that $\bigvee_{i<n} \sigma_{i}(\varphi \vee \psi)$ covers $C$.

Suppose that $\sigma \in \operatorname{Aut}_{L}(\mathfrak{C})$. Then $\sigma\left(\bigvee_{i<n} \sigma_{i}(\varphi \vee \psi)\right)=\bigvee_{i<n} \sigma\left(\sigma_{i}(\varphi \vee \psi)\right)$ also covers $C$. Since $\psi$ is non-generic, $\psi^{\prime}=\bigvee_{j<n} \sigma\left(\sigma_{j}(\psi)\right)$ is also non-generic and so is $\psi^{\prime} \vee \psi$.

Now we can apply Lemma Lemma 4.8.

Proposition 4.11. Let $a \in C$. Consider the partial type $q(x, y)=d_{\alpha}(x, y) \leq 1$. Then $q(x, a) \subseteq$ $L_{\alpha}(a)$ is generic and hence proper.

Proof. Suppose $\varphi(x, a)$ is a formula in $q(x, a)$. Suppose $\varphi$ is non-generic. This means that for every $n$ Lascar strong conjugates $a_{0}, \ldots, a_{n-1}$ of $a$, there is some $a^{\prime} \in C$ (so another Lascar conjugate of a) such that $\neg \varphi\left(a^{\prime}, a_{i}\right)$ holds for all $i<n$. Thus we can construct an infinite sequence $\left\langle a_{i} \mid i<\omega\right\rangle$ of Lascar conjugates of $a$ such that for every $i<\omega, \neg \varphi\left(a_{i}, a_{j}\right)$ holds for all $j<i$.

By Fact 1.10 (1), there is an indiscernible sequence $\left\langle b_{i} \mid i<\omega\right\rangle$ such that for all $j<i<\omega$, $\neg \varphi\left(b_{i}, b_{j}\right)$ holds. But this is a contradiction because by definition $d_{\alpha}\left(b_{1}, b_{0}\right) \leq 1$.

Theorem 4.12. Main Theorem $A$ holds:

Suppose $T$ is a complete countable first-order theory, $\alpha$ a countable ordinal, and suppose $Y$ is a pseudo $G_{\delta}$ subset of $\mathfrak{C}^{\alpha}$ which is closed under $\equiv_{L}^{\alpha}$. If for some $a \in Y,[a]_{\equiv_{L}^{\alpha}}$ is not d-bounded, then $\equiv_{L}^{\alpha} \uparrow Y$ is non-smooth.

Proof. Let $C=[a]_{\equiv_{L}^{\alpha}}$. For what follows when we write proper, we mean $C$-proper.

We want to apply Corollary 2.3 with $Y$ there being $Y_{M}$ (See Proposition 1.41) for some countable model $M$. Hence we will construct a pair $(M, p)$ such that $M$ is a countable model of $T$ and $\mathbf{x}=p \in Y_{M}$ satisfies the condition in Corollary 2.3. Translating, this means that for every formula $\varphi \in p$, and every $N<\omega$, there exists some Lascar strong automorphism $\sigma$ such that $\sigma(M)=M$, $\varphi \in \sigma(p)$ and $d_{\alpha}(\sigma(p), p)>N$.

Let $q(x)=d_{\alpha}(x, a) \leq 1$ (as in Proposition 4.11). We construct a sequence $\left\langle\sigma_{i}, p_{i}, M_{i} \mid i<\omega\right\rangle$ such that:

(1) $M_{i}$ is a finite set for all $i<\omega$.

(2) $M_{i} \subseteq M_{i+1}$ and $p_{i} \subseteq p_{i+1}$ for all $i<\omega$.

(3) For all $i<\omega, p_{i}$ is a finite type over $M_{i}$ such that $p_{i} \cup q$ is proper.

(4) For all $i<\omega, \sigma_{i}$ is a Lascar strong automorphism.

(5) For every $i<\omega$ and formula of $\varphi \in L_{1}\left(M_{i}\right)$, if $\varphi$ is not empty then for some $i<j<\omega$ there is some $c \in M_{j}$ such that $\varphi(c)$ holds. 
(6) For every $i<\omega$ and $n<\omega$ there exists some $i<j<\omega$ such that $M_{j}$ contains $\bigcup_{-n<l<n, i^{\prime}<i} \sigma_{i^{\prime}}^{(l)}\left(M_{i}\right)$.

(7) For every $i<\omega$ and formula $\varphi \in L_{\alpha}\left(M_{i}\right)$, there is some $i<j<\omega$ such that $p_{j}$ contains either $\varphi$ or $\neg \varphi$.

(8) For every $i<\omega, N<\omega$ and $\varphi \in p_{i}$ there are some $i<j<j^{\prime}<\omega$ such that $d_{\alpha}\left(\sigma_{j}(a), a\right)>$ $N$ and $\sigma_{j}^{-1}(\varphi) \in p_{j^{\prime}}$.

If we succeed, then let $M=\bigcup M_{i}, p=\bigcup p_{i} . M$ is a model by (5), and $p \in S(M)$ and even belongs to $Y_{M}$ by (7) and (3).

(3), (6) and (8) imply that $(M, p)$ satisfy the required condition: for every formula $\varphi(x) \in p$ (i.e., $p \in[\varphi]$ ) and $N<\omega$, there is some $\sigma_{j}$ as in (8). By (3), $d_{\alpha}(c, a) \leq 3$ for any $c \models p$ (because there exists some $c^{\prime} \models p \cup q$, and $\left.d_{\alpha}\left(c, c^{\prime}\right) \leq 2\right)$. So $d_{\alpha}\left(c, \sigma_{j}(a)\right)>N-3$. By (6) $\sigma_{j}(M)=M$, and so $d_{\alpha}\left(p, \sigma_{j}^{*}(p)\right)$ is well defined and $>N-6$ (as for any $c \models \sigma_{j}^{*}(p), d_{\alpha}\left(c, \sigma_{j}(a)\right) \leq 3$ ). Finally, $\varphi \in \sigma_{j}^{*}(p)$.

The construction:

Let $M_{0}$ and $p_{0}$ be $\emptyset$. Note that condition (3) holds by Proposition 4.11.

Now we partition the work so that can satisfy all conditions. In each stage we take care of one of $(5)-(8)$.

(5) and (6) are easy (just add some elements to $M_{i}$ ). (7) can be achieved by Lemma 4.9.

For (8) we need some argument. So suppose we are in stage $i+1$ of the construction and we deal with (8), i.e., we are given $N<\omega$ and $\varphi \in p_{i}$. By Proposition 4.10, there are $\tau_{0}, \ldots \tau_{n-1} \in$ Aut $f_{L}(\mathfrak{C})$ such that for every $\sigma \in$ Aut $f_{L}(\mathfrak{C})$, there exists some $j<n$ such that $q \cup p_{i} \cup\left\{\sigma\left(\tau_{j}(\varphi)\right)\right\}$ is proper. There is some bound $k$ on $\tau_{j}$ for all $j<n$. Let $\sigma \in \operatorname{Aut} f_{L}(\mathfrak{C})$ be such that $d_{\alpha}(a, \sigma(a))>N+k$. By the triangle inequality, $d_{\alpha}\left(a, \sigma\left(\tau_{j}(a)\right)\right)>N$ for all $j<n$. For some $j<n, q \cup p_{i} \cup\left\{\sigma\left(\tau_{j}(\varphi)\right)\right\}$ is proper, so let $\sigma_{i+1}=\left(\sigma \circ \tau_{j}\right)^{-1}$ (note that $d_{\alpha}\left(a,\left(\sigma \circ \tau_{j}\right)(a)\right)=$ $\left.d_{\alpha}\left(a,\left(\sigma \circ \tau_{j}\right)^{-1}(a)\right)\right)$ and $p_{i+1}=p_{i} \cup\left\{\sigma\left(\tau_{j}(\varphi)\right)\right\}$ and continue.

\section{THE GENERAL CASE}

Here we adapt our techniques to the case where the language is not necessarily countable.

Theorem 5.1. Main theorem B holds:

Suppose $T$ is a complete first-order theory, $\alpha$ a small ordinal. Suppose $Y \subseteq \mathfrak{C}^{\alpha}$ is closed under $\equiv_{L}^{\alpha}$ and for some $a \in Y,[a]_{\equiv_{L}^{\alpha}}$ is not d-bounded. Suppose $Y$ is pseudo strong Choquet. Then there is a model $M$ of size $|T|+|\alpha|$ and a function $\phi: 2^{\omega} \rightarrow \mathcal{P}\left(Y_{M}\right)$ such that for every $y, z \in 2^{\omega}$ :

- $\phi(y)$ is a non-empty closed $G_{\delta}$ subset of $Y_{M}$.

- If $z \mathbb{E}_{0} y$ then there is a some $\gamma \in \Gamma$ such that $\gamma \cdot \phi(z)=\phi(y)$.

- If $\sim z \mathbb{E}_{0} y$ then $(\phi(y) \times \phi(z)) \cap \equiv_{L}^{\alpha, M}=\emptyset$. 
In particular, $\left|Y / \equiv_{L}^{\alpha}\right| \geq 2^{\aleph_{0}}$.

Proof. The idea is to simultaneously construct a countable language $L^{\prime}$, a countable model $M^{\prime}$, a countable sub-tuple of the first $\alpha$ variables, an $L^{\prime}$-type over $M^{\prime}$ in these variables and a countable group of Lascar strong automorphisms so that we can apply Corollary 2.6. Eventually, $\mathbf{x}$ will be any completion of the $L^{\prime}$-type over $M^{\prime}$ to a complete $L$-type over $M$.

So we will need a more elaborate argument than the one used in Theorem 4.12 that will also use the proof of Lemma 2.7 (but not the lemma itself). That is, we try to construct the winning strategy along with the model and language.

Let $C=[a]_{\equiv_{L}^{\alpha}}$. For what follows when we write proper, we mean $C$-proper. Fix a countable set $S$ of Lascar strong automorphisms that witness that $C$ is not $d$-bounded, i.e., such that for all $N>0$, there is some $\sigma \in S$ such that $d_{\alpha}(a, \sigma(a))>N$.

Let $M$ be a model of $T$ of size $|T|+|\alpha|$ that contains $a$ such that every $\sigma \in S$ fixes $M$ setwise and for every generic formula over $M$, there are Lascar strong automorphisms that witness it which fix $M$ setwise. Such a model can be constructed as in Claim 3.1. Let $\Gamma$ be the group of Lascar strong automorphisms that fix $M$ setwise. Let $S t$ be a strategy for player B that witnesses that $Y_{M}$ is strong Choquet.

We construct:

- A countable sub-language $L^{\prime} \subseteq L$.

- A countable model $M^{\prime} \prec M \uparrow L^{\prime}$.

- A countable sub-tuple $x^{\prime}$ of the first $\alpha$ variables. For notational simplicity we will assume that $x^{\prime}$ is the first $\beta$ variables for a countable ordinal $\beta$.

- A complete $L^{\prime}$-type $p$ over $M_{0}$ in $x^{\prime}$ which is consistent with the type $q(x)=d_{\alpha}(x, a) \leq 1$.

- A countable subgroup $\Sigma \subseteq \Gamma$ of automorphism that fix $M^{\prime}$ and $M$ setwise.

- A countable set $Q$ of complete types in $S_{\alpha}(M)$ contained in $Y_{M}$.

Such that:

(1) For every formula $\varphi \in p$ and natural number $N$, there is an automorphism $\sigma \in \Sigma$ such that $\sigma^{-1}(\varphi) \in p$ and $d_{\beta}^{\prime}\left(\sigma^{*}(p), p\right)>N$ where $d^{\prime}$ is the Lascar metric when restricted to $L^{\prime}$.

(2) For every $\sigma_{0}, \ldots, \sigma_{n} \in \Sigma, r_{0}, \ldots, r_{n-1} \in Q$ and every sequence of $L_{\beta}^{\prime}\left(M^{\prime}\right)$ formulas $\left\langle\left(\varphi_{i}, \psi_{i}\right) \mid i<n\right\rangle$ and a formula $\varphi_{n}$ such that:

(a) $\varphi_{i+1} \vdash \psi_{i} \vdash \varphi_{i}$ for all $i<n$.

(b) $\psi_{i} \in \sigma_{i}^{*}(p)$ (in other words, $\sigma_{i}^{*}(p)$ is in the open set $\left[\psi_{i}\right]$ ).

(c) $r_{i}$ is a complete extension of $\left\{\varphi_{i}\right\}$ consistent with $\sigma_{i}^{*}(q)$.

(d) $\varphi_{n} \in \sigma_{n}^{*}(p)$. 
(e) For each $i<n, \psi_{i}$ is such that $\left[\psi_{i}\right] \cap Y_{M}$ is a basic open subset of player B's move according to $S t$ in the strong Choquet game where player A plays $\varphi_{i}$ and $r_{i}$.

There is a type $r_{n} \in Q$ containing $\varphi_{n}$ and a formula $\psi_{n}$ in $\sigma_{n}^{*}(p) \cap r_{n}$ contained in $\varphi_{n}$ which is a subset of player B's move according to $S t$ in the strong Choquet game described in (e) where in the $n$ 'th move player A chooses $\varphi_{n}$ and $r_{n}$.

For the construction we repeat the proof of Theorem 4.12 inside $M$. As there, we let $q=d(x, a) \leq$ 1 , and note that it is proper. The differences are:

* We choose our automorphisms from $\Gamma$ (this is no problem, since they all come from witnesses of genericity of certain formulas over $M$ composed with an element from $S$ by the proof of 4.10$)$.

* We have to take care of $d^{\prime}$ instead of $d$. So in (8) there we increase the language $L^{\prime}$ so that not only $d_{\alpha}\left(\sigma_{j}(a), a\right)>N$ is true in $L$, but it also true in $L^{\prime}$. Similarly add some variables to the tuple of variables we construct so that this remains true when restricted to these variables.

* We add a step to the construction that makes sure that the set of automorphisms is a group.

$\star$ For (2), we add a step to the construction. We have to take care of every choice of $\sigma_{0}, \ldots, \sigma_{n} \in \Sigma, r_{0}, \ldots, r_{n-1}$, a formula $\varphi_{n}$ and a sequence of $L_{\beta}^{\prime}\left(M^{\prime}\right)$ formulas $\left\langle\left(\varphi_{i}, \psi_{i}\right) \mid i<n\right\rangle$ from the language and model constructed thus far that satisfy (a)-(e) above. We may assume that $\varphi_{n}$ is the conjunction of $\sigma_{n}$ applied to the current finite partial type we have. For every complete extension $r \in S_{\alpha}(M)$ of $\left\{\varphi_{n}\right\}$ consistent with $\sigma_{n}^{*}(q)$, there is some open set $r \in V_{r} \subseteq\left[\varphi_{n}\right] \cap Y_{M}$ that player B plays according to $S t$ in the strong Choquet game described in (e) where in the $n$ 'th move player A chooses $\varphi_{n}$ and $r$. Let $\psi_{r}$ be a formula in $L_{\alpha}(M)$ that contains $r$ (i.e., $\left.\psi_{r} \in r\right), \psi_{r} \vdash \varphi_{n}$ and $\left[\psi_{r}\right] \cap Y_{M}$ is contained in $V_{r}$. It follows that $\left\{\varphi_{n}\right\} \cup \sigma_{n}^{*}(q) \vdash \bigvee \psi_{r}$. By compactness and by Lemma 4.9 for some $r$, $\sigma_{n}^{*}(q) \cup\left\{\varphi_{n}, \psi_{r}\right\}$ is proper. So we may add $\sigma_{n}^{-1}\left(\psi_{r}\right)$ to our partial type. Also we add the symbols appearing in $\psi_{r}$ to the language and the variables appearing in it to the tuple of variables. Finally, add $r$ to $Q$.

When the construction is done, it is easy to see that letting $\mathbf{x}$ be any completion of the complete $L_{\beta}^{\prime}\left(M^{\prime}\right)$ type constructed $p$, it satisfies all the demands of Corollary 2.6. For instance, we need to check that with the topology induced on $Y_{M}$ by $L^{\prime}, M^{\prime}$, and $\beta, Y$ is strong Choquet over $\Sigma \cdot \mathbf{x}$. The point is that if player A chooses some basic open set $[\varphi]$ containing $\sigma^{*}(\mathbf{x})$ for some $\sigma \in \Sigma$, then by construction there is some formula $\psi$ in $\sigma^{*}(p)$ (so $[\psi]$ contains $\sigma^{*}(\mathbf{x})$ ) that is contained in $[\varphi]$ and some type $r_{0} \in Q$ such that $\psi$ is contained in player B's response to $\left([\varphi], r_{0}\right)$. So player B will now choose $[\psi] \cap Y_{M}$. So we simulate a game in $Y_{M}$ in which player A chooses types from $Q$, 
and player B responds by choosing a basic open subset of what $S t$ says. Since $S t$ was a winning strategy, the intersection must be nonempty.

\section{REFERENCES}

[BK96] Howard Becker and Alexander S. Kechris. The descriptive set theory of Polish group actions, volume 232 of London Mathematical Society Lecture Note Series. Cambridge University Press, Cambridge, 1996.

[Cas11] Enrique Casanovas. Simple theories and hyperimaginaries, volume 39 of Lecture Notes in Logic. Association for Symbolic Logic, Chicago, IL, 2011.

[CLPZ01] E. Casanovas, D. Lascar, A. Pillay, and M. Ziegler. Galois groups of first order theories. J. Math. Log., $1(2): 305-319,2001$.

[HKL90] L. A. Harrington, A. S. Kechris, and A. Louveau. A Glimm-Effros dichotomy for Borel equivalence relations. J. Amer. Math. Soc., 3(4):903-928, 1990.

[Hod93] Wilfrid Hodges. Model Theory, volume 42 of Encyclopedia of mathematics and its applications. Cambridge University Press, Great Britain, 1993.

[Hru98] Ehud Hrushovski. Simplicity and the lascar group. 1998.

[Kec95] Alexander S. Kechris. Classical descriptive set theory, volume 156 of Graduate Texts in Mathematics. Springer-Verlag, New York, 1995.

[Ker07] Aviv Keren. Equivalence relations \& topological automorphism groups in simple theories. Master's thesis, Hebrew University of Jerusalm, Israel, 2007.

[KPS12] Krzysztof Krupinski, Anand Pillay, and Slawomir Solecki. Borel equivalence relations and lascar strong types. 2012. arXiv:1204.3485

[New03] Ludomir Newelski. The diameter of a Lascar strong type. Fund. Math., 176(2):157-170, 2003.

[NP06] Ludomir Newelski and Marcin Petrykowski. Weak generic types and coverings of groups. I. Fund. Math., 191(3):201-225, 2006.

[Pel08] Rodrigo Peláez Peláez. About the Lascar Group. PhD thesis, Universitat de Barcelona, Departament de Lògica, Història i Filosofia de la Ciència, 2008.

[Sil80] Jack H. Silver. Counting the number of equivalence classes of Borel and coanalytic equivalence relations. Ann. Math. Logic, 18(1):1-28, 1980.

[TZ12] Katrin Tent and Martin Ziegler. A Course in Model Theory (Lecture Notes in Logic). Cambridge University Press, 2012. 